\title{
Hybrid integration on low-cost flex foils using photonic flash soldiering
}

\author{
Saeed Khoshfetrat Pakazad ${ }^{1}$, Marco Barink ${ }^{1}$, Gari Arutinov ${ }^{1}$, Rob Hendriks ${ }^{2}$, Roel Kusters ${ }^{1}$, Maarten Cauwe ${ }^{3}$ and Jeroen van \\ den Brand ${ }^{1}$ \\ ${ }^{1}$ Holst Centre / TNO, High Tech Campus 31, 5656AE, Eindhoven, The Netherlands \\ ${ }^{2}$ NovaCentrix, 400 Parker Rd 1110, Austin, TX 78728, United States \\ ${ }^{3}$ Advanced Packaging Centre for Microsystems Technology (CMST) IMEC vzw Technologiepark 15 \\ B-9052 Zwijnaarde, Belgium \\ E-mail : saeed.pakazad@tno.nl
}

\begin{abstract}
Soldering of packaged electronic components using industry standard $\mathrm{Sn}-\mathrm{Ag}-\mathrm{Cu}$ (SAC) lead-free solders on low-cost foils, which are often the substrate of choice for flexible electronics, is challenging. This is mainly originating from the fact that the reflow temperatures of these solder alloys are normally higher than the maximum processing temperature of the low-cost flex foils. To enable component integration on the low-cost foils a novel method for soldering has been introduced by Holst Centre as an alternative to oven reflow, termed "photonic soldering". In this method high intensity photonic flashes are used to deliver the thermal energy required for soldering. By taking advantage of the selectivity of light absorption, the required energy for soldering is delivered to the components and circuit tracks while excessive heating of the foils is avoided. This paper presents successful photonic flash soldering of packaged LED components on low-cost polyethylene terephthalate (PET) foils using conventional SAC solders as a demonstration of the capabilities of this novel soldering technology.
\end{abstract}

\section{Introduction}

Reliable integration of conventional electronic chips and passive components next to printed electronics on flexible foils enables realization of smart flexible systems with complex functionality $[1,2]$. To reduce the cost of such systems, low-cost flexible foils are preferred as the base substrate. However, reliable bonding of components on low-cost foils is generally challenging, due to limited processing temperature range of the foils. Conductive adhesives with relatively low curing temperatures (typically $120-150{ }^{\circ} \mathrm{C}$ ) are commonly used for component bonding on such foils [3-5]. While for a variety of applications conductive adhesives provide sufficient reliability, for more demanding applications such as automotive or wearables higher quality bonds are required.

Soldering has been commonly used in electronic industry for reliable component bonding on printed circuit boards (PCBs). However, the melting temperature of industry standard solder alloys such as lead-free Sn-Ag$\mathrm{Cu}$ (SAC) is above the maximum processing temperature range of low-cost foils such as polyester foils, which renders oven solder reflow on these foils practically impossible. Low-temperature solder alloys with melting temperatures below $150{ }^{\circ} \mathrm{C}$ such as $\mathrm{SnBi}$ alloys can be used as alternatives to SAC solders; however there are reliability challenges associated with low-temperature solders which makes them less suitable for certain applications [6, 7].

By taking advantage of the optical transparency of the polyester foils, it is possible to selectively heat the components and circuit tracks to temperatures sufficient for soldering using high-intensity light pulses while preventing excessive heating of the foils. In this method called "photonic soldering", intense light pulses from a flash lamp illuminate the area of interest, the components and circuit tracks absorb the incident light energy causing a fast temperature increase, while the transparent foil passes through most of the incident light energy. The amount of temperature increase in a component depends on the heat capacity of the component and the amount of absorbed light by the component. As a result, components with different sizes, packaging materials and surface characteristics show different levels and time scales of heating with the same incident photonic energy [8, 9]. In order to enable soldering of a variety of component types on the same foil, the illumination can be performed through a filtering mask which tunes the amount of energy delivered to each component type [10].

Previously, successful photonic soldering of bare-die thin silicon chips on flex foils have been demonstrated [8$10]$. In these experiments chips with thicknesses ranging from 20 to $150 \mu \mathrm{m}$ were soldered with photonic pulse energies roughly ranging from 2 to $6 \mathrm{~J} / \mathrm{cm}^{2}$. In each case the soldering energy was delivered by a single light pulse with a fixed duration of several milliseconds, while the amount of energy was varied by changing the discharge voltage of a xenon flash lamp.

In photonic soldering the light energy absorbed on the top surface of the component needs to diffuse through the bulk of the component to increase the temperature of the components, solder paste and the contact pads to a level sufficient to initiate melting of the solder alloy. The relatively high thermal conductivity and low thermal capacity of the thin bare-die silicon chips, enables photonic flash soldering at very short time scales and relatively low pulse energies as reported before [8-10]. For packaged components, however, the mold epoxy and other polymers used in the packaging, normally have orders of magnitude lower thermal conductivity compared to silicon [11]. This combined with the fact that packaged components are generally thicker and bulkier than baredie chips, makes photonic soldering of such components more challenging. Therefore, the photonic energy delivery scheme needs to be adapted for such packaged components. 
In this paper, this technological challenge is addressed by focusing on photonic soldering of packaged LED components on low-cost flex foils. By extending the time scale of photonic energy delivery, packaged LEDs are successfully soldered on copper clad polyethylene terephthalate (PET) foils using a commercial lead-free SAC solder paste. The demonstrated technology can enable production of reliable low-cost large area lighting solutions amongst other flexible electronic systems.

\section{Materials and methods}

The LED components used in the experiments were SML-P1 PICOLED series (ROHM semiconductor) with $1006(0402)$ footprint, $1.0 \mathrm{~mm} \times 0.6 \mathrm{~mm}$ in dimension and $0.2 \mathrm{~mm}$ in thickness. As shown in Fig. 1a, the wire-bonded silicon chip constitutes a small fraction of the packaged component. The chip is covered with a polymeric transparent lens. These LEDs were particularly selected for their small form factor and thin packaging, which makes them an interesting candidate for expanding the capabilities of photonic soldering. The PET flex foils were $50 \mu \mathrm{m}$ in thickness, with a 13 - $\mu \mathrm{m}$-thick laminated copper layer using an intermediate $13-\mu \mathrm{m}$-thick layer of polyurethane (PU) laminating adhesive. The foils were supplied by Imec CMST, Belgium. The solder paste used was a lead-free Amtech NC-560-LF 96.5Sn/3.0Ag/0.5Cu alloy (Inventech, USA) with type 6 size and $85 \%$ solid content. The solder paste was applied on the copper circuitry by using a $25-\mu \mathrm{m}$-thick stainless steel stencil. After solder paste deposition the components were placed on the foils using a Dr. Tresky T3200 semiautomatic pick and place machine (Dr. Tresky, Switzerland).

The equipment used for the photonic soldering experiments was a PulseForge 1300 xenon lamp system (Novacentrix, Austin, USA). In this machine a capacitor bank charged to an adjustable voltage of several hundred volts is discharged over a xenon lamp through a switch, generating high intensity light pulses. The switch can be programmed through the machine interface software in order to generate single pulses with certain durations or a train of pulses with adjustable on-off periods. The energy delivered by each pulse can be tuned by adjusting the charging voltage of the capacitor bank and the duration of the pulse. The actual delivered energy was measured with the machine's integrated bolometer. For the experiments the foils were placed on the steel chuck of the machine 6 $\mathrm{mm}$ bellow the protective window of the lamp. The foils were fixed to a glass plate using a double-sided tape to prevent movement due to thermal expansion which can cause component displacement during photonic exposures. After component placement, the foils were pre-heated in a convection oven at $95{ }^{\circ} \mathrm{C}$ for 2 minutes and cooled down to room temperature prior to each photonic exposure. This is to allow the solvent in the solder paste to evaporate before photonic exposure, otherwise the steep temperature increase within the short duration of a photonic pulse can cause burst evaporation of the solvent resulting in component displacement.

Different time scales of energy delivery were investigated in the experiments, starting with our previously reported exposure schemes [8-10], and further prolonging the energy delivery time frame using pulse trains to improve the soldering performance for the packaged LED components.
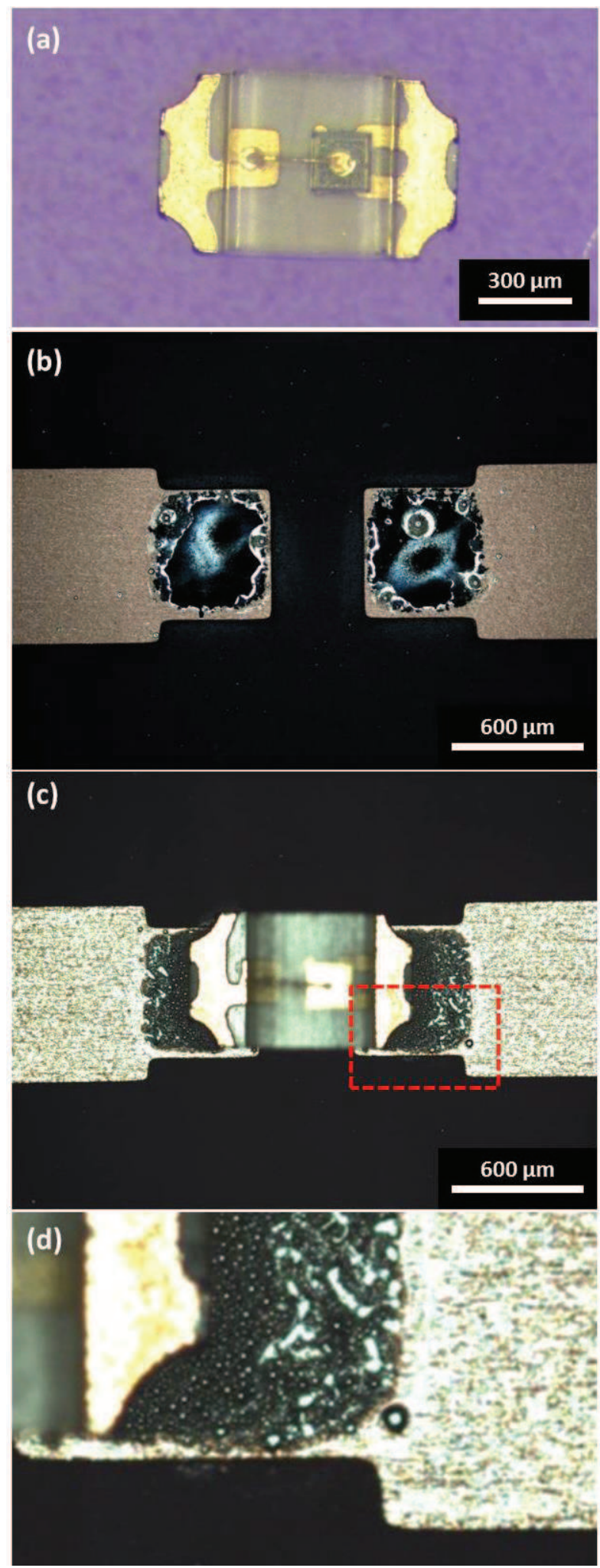

Figure 1. (a) Optical micrograph of the LED component. Optical micrographs of the contact pads, each exposed with a photonic pulse with $5 \mathrm{~ms}$ duration and $6 \mathrm{~J} / \mathrm{cm}^{2}$ energy: (b) without the component placed, the applied solder paste melts completely, however, (c) with the component present melting of the solder paste is barely initiated. (d) Magnified image of the region denoted in c. 


\section{Results and discussion}

For a successful soldering process the contact pads of the component and the copper circuitry should be both heated sufficiently to allow for proper wetting and intermetallic formation. The amount of energy as well as the time scale during which the energy is delivered, both play an important role in an efficient heating scheme. For packaged components due to orders of magnitude lower thermal conductivity of the packaging material compared to bare silicon chips and the copper circuitry, a short time scale for the photonic pulse will cause only superficial heating of the components, and before the heat can diffuse to the component's bulk, it is dissipated through convective and radiative mechanisms. This effect is demonstrated in Fig. 1 which shows the results of two experiments with and without a LED component using the same photonic pulse settings. In the experiment without the LED component placed (Fig. 1a) the incident energy is sufficient to completely melt the deposited solder paste. It is noteworthy that with the same pulse settings the energy is also sufficient to solder $150 \mu \mathrm{m}$ thick bare-die silicon chips as previously demonstrated [10]. However, with the LED component in place using the same exposure, the soldering is barely initiated near the component (Fig. 1b\&c), as a result of insufficient heating due to component size and low thermal conductivity of the packaging material as explained before.

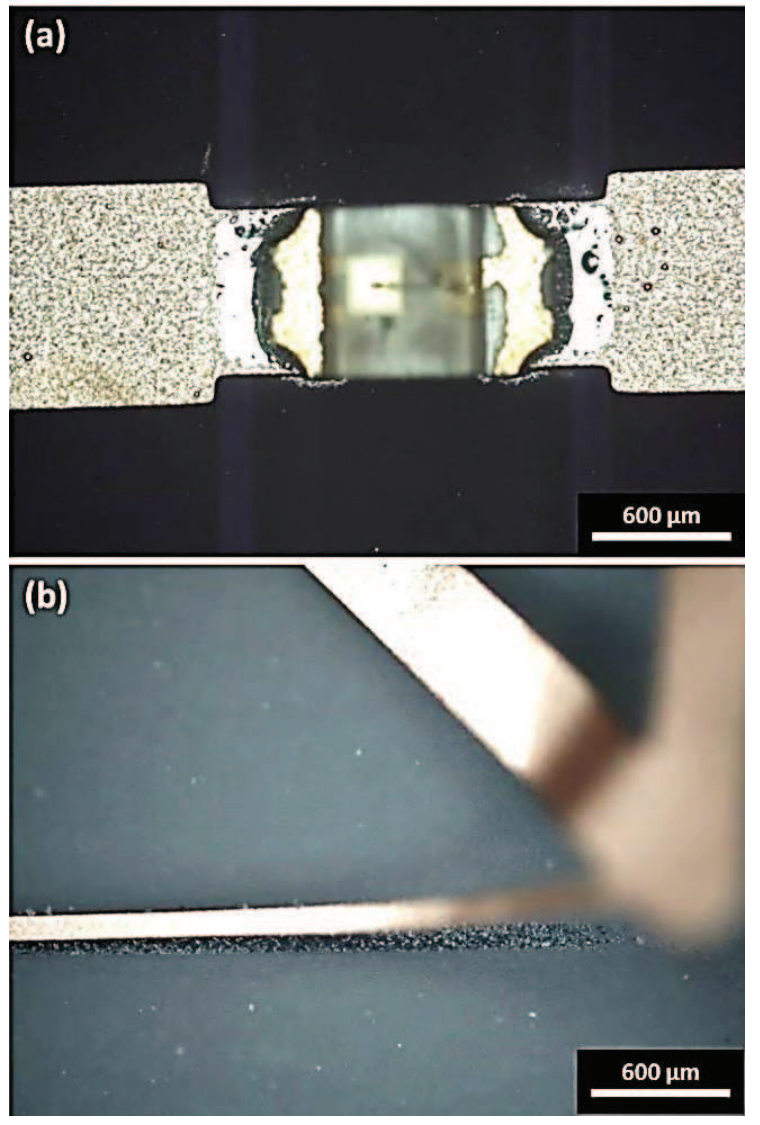

Figure 2. Optical micrographs of (a) the component and contact pads, after exposure with a photonic pulse with $5 \mathrm{~ms}$ duration and $7 \mathrm{~J} / \mathrm{cm}^{2}$ energy, and (b) copper tracks delamination and out-of-plane bending after exposure (top view).
Keeping the duration of the pulse the same while increasing the energy of the pulse from $6 \mathrm{~J} / \mathrm{cm}^{2}$ to $7 \mathrm{~J} / \mathrm{cm}^{2}$ results in increased but yet incomplete melting of the solder paste (Figs. 2a \& 3a). As shown in Fig. 3a in the vicinity of the component and on the side-walls the solder is still not completely melted, indicating insufficient heating of the component, while on the adjacent copper tracks the same incident energy is sufficient to melt the solder.

Further increasing the energy of the pulse with the same pulse duration is not possible due to delamination of the copper tracks from the foil which is already initiated at $7 \mathrm{~J} / \mathrm{cm}^{2}$ energy (Fig. 2b). The copper delamination can be attributed to excessive heating and thermal degradation of the underlying adhesive layer as a result of the accumulation of thermal energy below the track originating from the combination of low thermal conductivity of the foil material with the short time scale of energy delivery.
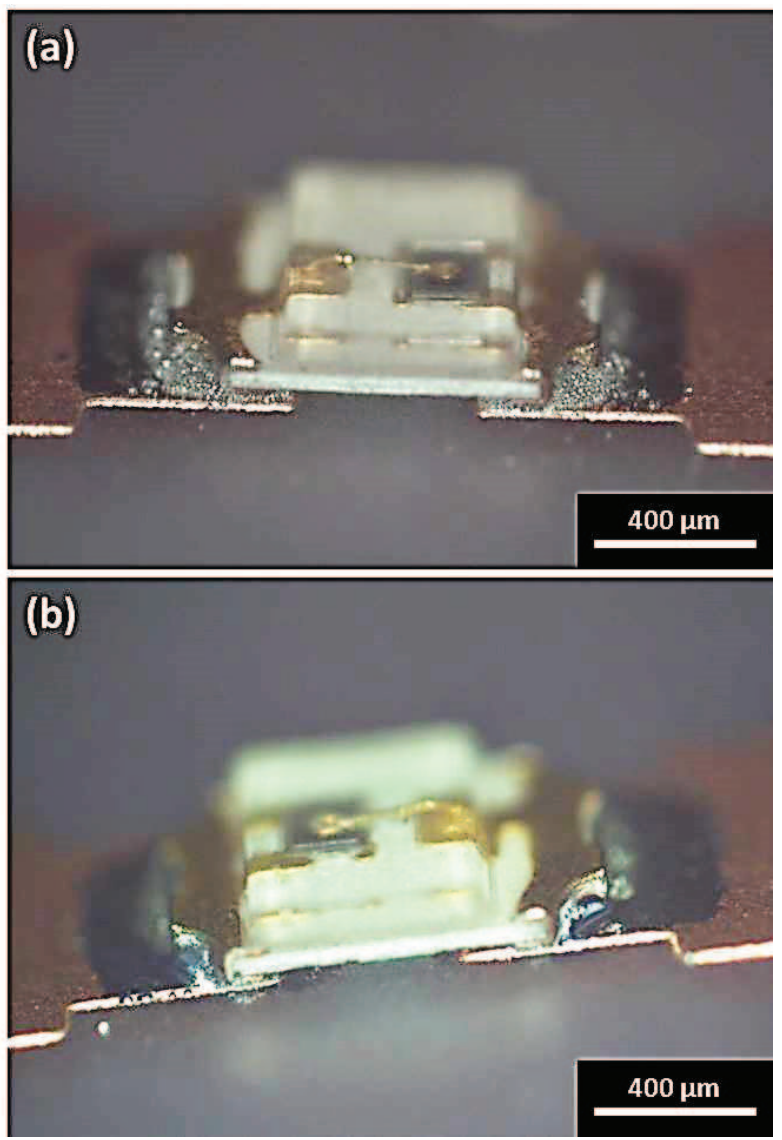

Figure 3. Optical micrographs at an angel of the components after exposure with, (a) a photonic pulse with $5 \mathrm{~ms}$ duration and $7 \mathrm{~J} / \mathrm{cm}^{2}$ energy, and (b) a modulated pulse train with $200 \mathrm{~ms}$ duration and $16 \mathrm{~J} / \mathrm{cm}^{2}$ energy.

In order to improve the heating dynamics, the energy can be delivered over a longer time frame to allow for sufficient time for heat diffusion, in order to avoid superficial over-heating. Since the light pulses are generated by a capacitive discharge over a xenon lamp extending the duration of a single pulse beyond approximately $10 \mathrm{~ms}$ is not possible, because the capacitor bank is almost completely depleted within this 
time frame. However, it is possible to on-off modulate the discharge in order to distribute the contained energy in the pulse over a longer time scale. The on and off periods of the modulated pulse can be fully adjusted in the software of the machine. Our experiments with different time scales showed that, distributing the energy over a time period of approximately $200 \mathrm{~ms}$ yields the proper heating dynamics for this type of foil and component. Since the on-off modulation introduces cooling periods in between consecutive pulses, the total energy of the pulse train should be increased to account for the dissipated energy. Fig. $3 \mathrm{~b}$ shows the result of photonic soldering with a $200 \mathrm{~ms}$ modulated pulse with $16 \mathrm{~J} / \mathrm{cm}^{2}$ energy. It can be observed that the solder paste is properly melted forming a meniscus in this case compared to the case soldered with the $5 \mathrm{~ms}$ pulse shown in Fig. 3a. With the modulated pulse, no delamination of the copper tracks is observed, indicating that in this case proper heating dynamics prevents excessive heating of the adhesive layer below the tracks as described before.

After photonic soldering, the functionality of the LEDs was not compromised and no defects were observed under optical microscopy inspection (Fig. 4). However, further investigation of the effects of high temperature gradients developed during photonic soldering across components, with different types of packaging, on the reliability of the components is required.

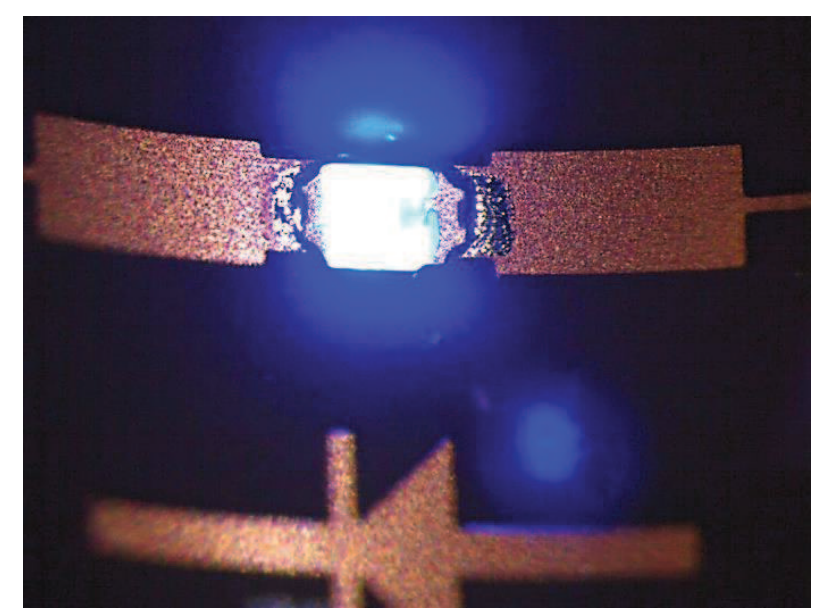

Figure 4. Optical micrograph of a powered LED after photonic soldering on a PET flex foil using a 200 ms modulated pulse with $16 \mathrm{~J} / \mathrm{cm}^{2}$ energy.

\section{Conclusions}

This paper demonstrated the use of photonic soldering technology to integrate packaged LED components on copper clad low-cost PET foils. By using high intensity photonic pulses, it was shown that component integration can be accomplished using industry standard lead-free SAC solder alloys with a eutectic point higher than the maximum processing temperature of the foils.

By adjusting the rate of energy delivery to the system, a heating dynamics was achieved which enables effective soldering of the LEDs while preventing thermal damage to the foils and delamination of the copper circuitry. Further investigation of the metallurgical properties of the formed bonds as well as reliability tests using humidity and temperature cycling is required to assess the quality of the photonically soldered bonds compared to that of other conventional methods. The efficiency of soldering can be further optimized and adapted for multiple component types by tuning the on-off periods and energy of the modulated pulse train assisted with relevant thermal simulation. Additionally, the constraints on the components' dimensions and packaging types suitable for photonic soldering should be determined in the future.

The presented results show the potential of photonic soldering as an integration method on low-cost foils using industry standard lead-free solder alloys providing in principle higher reliability than that of conductive adhesives and low-temperature solders in a roll-to-roll compatible process.

\section{References}

[1] A. Nathan, A. Ahnood, M. T. Cole, S. Lee, Y. Suzuki, P. Hiralal, et al., "Flexible Electronics: The Next Ubiquitous Platform," Proceedings of the IEEE, vol. 100, pp. 1486-1517, 2012.

[2] R. H. Reuss, G. B. Raupp, and B. E. Gnade, "Special issue on advanced flexible electronics for sensing applications [Scanning the Issue]," Proceedings of the IEEE, vol. 103, pp. 491-496, 2015.

[3] D. A. van den Ende, R. H. L. Kusters, M. Cauwe, A. van der Waal, and J. van den Brand, "Large area flexible lighting foils using distributed bare LED dies on polyester substrates," Microelectronics Reliability, vol. 53, pp. 1907-1915, 12// 2013.

[4] J. C. Jagt, "Reliability of electrically conductive adhesive joints for surface mount applications: a summary of the state of the art," IEEE Transactions on Components, Packaging, and Manufacturing Technology: Part A, vol. 21, pp. 215-225, 1998.

[5] S. A. Kudtarkar and J. E. Morris, "Reliability of electrically conductive adhesives," in Advanced Packaging Materials, 2002. Proceedings. 2002 8th International Symposium on, 2002, pp. 144150.

[6] K. Suganuma, "Advances in lead-free electronics soldering," Current Opinion in Solid State and Materials Science, vol. 5, pp. 55-64, 1// 2001.

$\mathrm{K}$. Zeng and K. N. Tu, "Six cases of reliability study of $\mathrm{Pb}$-free solder joints in electronic packaging technology," Materials Science and Engineering: R: Reports, vol. 38, pp. 55-105, 6/14/ 2002.

[8] D. A. v. d. Ende, R. Hendriks, R. Cauchois, R. H. L. Kusters, M. Cauwe, W. A. Groen, et al., "Photonic Flash Soldering of Thin Chips and SMD Components on Foils for Flexible Electronics," IEEE Transactions on Components, Packaging and Manufacturing Technology, vol. 4, pp. 1879-1886, 2014. 
[9] D. A. van den Ende, R. Hendriks, R. Cauchois, and W. A. Groen, "Large area photonic flash soldering of thin chips on flex foils for flexible electronic systems: In situ temperature measurements and thermal modelling," Electronic Materials Letters, vol. 10, pp. 1175 1183, 2014.

[10] G. Arutinov, J. v. d. Brand, and R. Hendriks, "Photonic Flash Soldering on Flex Foils for Flexible Electronic Systems," IEEE Electronic Components and Technology Conference, 2016.

[11] N. Kinjo, M. Ogata, K. Nishi, A. Kaneda, and K. Dušek, "Epoxy Molding Compounds as Encapsulation Materials for Microelectronic Devices," in Speciality Polymers/Polymer Physics, ed Berlin, Heidelberg: Springer Berlin Heidelberg, 1989, pp. 1-48. 